\title{
An advanced molecule-surface scattering instrument for study of vibrational energy transfer in gas-solid collisions
}

\author{
Qin Ran, Daniel Matsiev, and Alec M. Wodtke ${ }^{\text {a) }}$ \\ Department of Chemistry and Biochemistry, University of California, Santa Barbara, \\ California 93106-9510, USA \\ Daniel J. Auerbach \\ Gas Reaction Technologies, Inc., 861 Ward Drive, Santa Barbara, California 93111, USA
}

(Received 20 June 2007; accepted 19 September 2007; published online 15 October 2007)

\begin{abstract}
We describe an advanced and highly sensitive instrument for quantum state-resolved molecule-surface energy transfer studies under ultrahigh vacuum (UHV) conditions. The apparatus includes a beam source chamber, two differential pumping chambers, and a UHV chamber for surface preparation, surface characterization, and molecular beam scattering. Pulsed and collimated supersonic molecular beams are generated by expanding target molecule mixtures through a home-built pulsed nozzle, and excited quantum state-selected molecules were prepared via tunable, narrow-band laser overtone pumping. Detection systems have been designed to measure specific vibrational-rotational state, time-of-flight, angular and velocity distributions of molecular beams coming to and scattered off the surface. Facilities are provided to clean and characterize the surface under UHV conditions. Initial experiments on the scattering of $\mathrm{HCl}(v=0)$ from $\mathrm{Au}(111)$ show many advantages of this new instrument for fundamental studies of the energy transfer at the gas-surface interface. (C) 2007 American Institute of Physics. [DOI: 10.1063/1.2796149]
\end{abstract}

\section{INTRODUCTION}

Dynamics of molecule-surface interactions including energy transfer processes and chemical reactions have attracted great interest in a variety of experimental and theoretical fields such as heterogeneous catalysis, surface chemistry, semiconductor, and nanomaterial science in the past two decades. ${ }^{1-6}$ The use of molecular beam and laser techniques accompanied by surface preparation and characterization methods in surface science instruments ${ }^{1-3,7-9}$ has succeeded in probing many aspects of such interactions and aided in revealing dynamical pictures of energy flow and chemical reactions at the gas-solid interface. ${ }^{1-6}$ The collision of gas molecules with the surface is the initial step to any chemical reaction at a surface. The flow of energy into and out of chemical bonds during collisions is essential to bond rupture and formation. The study of vibrational energy transfer is taking an increasingly prominent place in this field. Vibrational excitation or de-excitation of gas molecules may result in surface phonon destruction or creation and/or electronic de-excitation or excitation. ${ }^{10-15}$ Vibrational motion of the gas molecules may influence trapping, ${ }^{14,16}$ exhibit isotope dependent effects, ${ }^{17,18}$ and play a vital role in vibrationally promoted surface reactions, ${ }^{14,19,20}$ electron transfer, ${ }^{21}$ and even electron emission. ${ }^{22}$ Much effort has been devoted to the study of electronically nonadiabatic influences in vibrational energy transfer at solid surfaces, but this has been accomplished for only a few molecule-surface systems.

Recently we designed and constructed a new molecule-

\footnotetext{
${ }^{\text {a) }}$ Author to whom correspondence should be addressed. Electronic mail: wodtke@chem.ucsb.edu
}

surface scattering instrument for systematic studies of vibrationally nonadiabatic energy transfer effects in gas-solid collisions. The main features of our new machine are similar to that which we used previously, ${ }^{21}$ but it has been greatly improved and optimized in several ways. A multichamber configuration has been chosen to allow preparation of molecular beams, characterization of the metal surface, and measurements of molecule-surface scattering carried out in different chambers. An optimized corrosion resistant pulsed valve and an intense, tunable, pulsed infrared laser system are used to generate high intensity state-selected molecular beams. This machine enables full measurements of the specific vibrational-rotational, temporal, angular, and velocity distributions of the incident and scattered molecules undergoing collisions with a surface over a broad range of kinetic energies and surface temperatures.

Our first experiments performed in this instrument involving $\mathrm{HCl}(v=0)$ scattered from $\mathrm{Au}(111)$ demonstrate the sensitivity and outstanding performance of this machine. We observed the first vibrationally inelastic scattering for $\mathrm{HCl}(v=0 \rightarrow 1)$ on a $\mathrm{Au}(111)$ surface $^{23,24}$ The present data show that vibrational excitation probabilities are in the range of $\sim 10^{-6}-10^{-4}$ and probabilities as low as $\sim 8 \times 10^{-7}$ could be observed. Such sensitivity represents a substantial improvement over the previous capability, allowing us to probe the variation of vibrational energy transfer over a much larger dynamic range for a wider variety of molecule-metal collision systems. We hope such results will lead to a detailed view of the fundamental dynamics at the atomic scale and provide a stringent test of theoretical understanding and computational models.

In this article we describe in detail the design and per- 


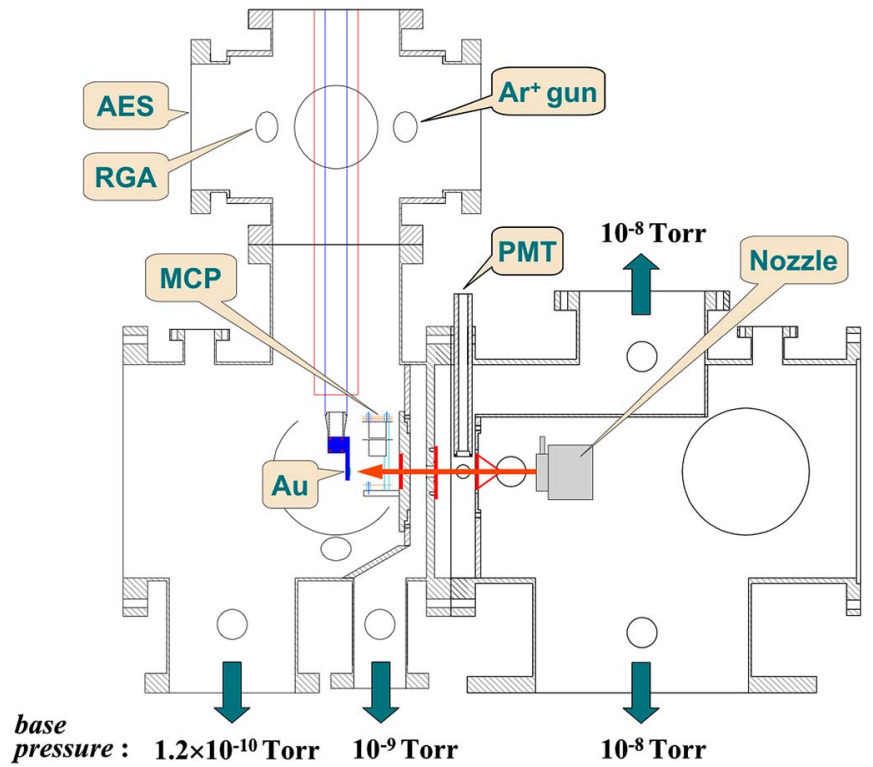

FIG. 1. (Color online) Schematic diagram of the advanced molecular beamsurface scattering apparatus. AES: Auger electron spectrometer; RGA: residual gas analyzer; MCP: microchannel plate; PMT: photomultiplier tube.

formance of a new advanced molecule-surface scattering instrument and present some preliminary results of $\mathrm{HCl}(v=0 \rightarrow 0,1)$ inelastic scattering from Au that exemplify the capabilities of this new apparatus.

\section{APPARATUS DESCRIPTION}

\section{A. Overview}

Figure 1 shows a schematic diagram of the new machine. It consists of four vacuum chambers (from right to left): source chamber, first differential chamber, second differential chamber, and a UHV surface science chamber. One of the important features in the design is the use of two differential pumping regions, which allows for production of intense pulsed or continuous molecular beams in one part of the machine operating at pressures up to $5 \times 10^{-5}$ Torr, while maintaining UHV $\left(<2 \times 10^{-9}\right.$ Torr $)$ in the scattering zone inside the surface chamber to reduce the effect of the source background.

An interesting aspect of the design is that the source chamber is equipped with a cryopump (ASC, Cryo Plex-10) driven by a helium compressor (ASC, M450). This improves source chamber pumping speed dramatically, especially for condensable gases. For example, the pumping speed for water, hydrogen, and air are 9000, 5000, and $3000 \mathrm{l} / \mathrm{s}$, respectively. In addition, the source chamber consists of a homebuilt piezoelectric pulsed valve, which we designed to render remarkably short molecular beam pulses. It is important to note that cryopumping cannot be used with a very common carrier gas $\mathrm{He}$, but for most applications, using $\mathrm{H}_{2}$ provides a perfectly adequate He substitute. The source region is connected to the first differential pumping region through a $1.5 \mathrm{~mm}$ diameter, sharp edged conical skimmer (Beam Dynamics). The first and second differential pumping chambers are pumped by 400 and $120 \mathrm{l} / \mathrm{s}$ turbomolecular pumps (Osaka, TG403 and TF160CA), respectively. These two re- gions are connected together by a small slide gate valve with a small aperture (3 $\mathrm{mm}$ diameter when fully open), which is designed to isolate the UHV surface chamber from the source chamber. The O-ring in the gate valve is still differentially pumped from the second differential pumping chamber. An optical collection system consisting of two focusing lenses resident in the first differential pumping chamber can be employed with a photomultiplier tube (PMT) to collect laser induced fluorescence from the beam. Three optical access ports, residing in the source, the first differential pumping and the surface chambers, respectively, are available for introduction of a laser beam. The UHV surface science chamber is pumped by a $3501 / \mathrm{s}$ turbomolecular pump (Leybold, NT360) which is backed by a second 70 1/s turbo pump (Pfeiffer-Balzers, TPU062) followed by a mechanical pump. The second differential pumping chamber and the surface chamber are connected by a $2 \mathrm{~mm}$ diameter aperture. This combination of improved pumping system and short pulsed valve design leads to excellent operating conditions. The base pressure of the UHV chamber is less than 1.2 $\times 10^{-10}$ Torr. With the pulsed molecular beam running at $10 \mathrm{~Hz}$, the pressure rises to $\sim 1 \times 10^{-9}$ Torr.

The UHV surface chamber has a two-level construction: the upper level, for surface preparation and characterization, and the lower one, used for molecular beam scattering. The upper section is equipped with an argon ion gun (LK Technologies, NGI3000), an Auger electron spectrometer (Physical Electronics, Ф15-255G), a UHV leak valve (Vacuum Generator), as well as a mass spectrometric residual gas analyzer (SRS, RGA-200). A UHV manipulator (Vacuum Generator Fisons Instruments, Omniax 800) with $800 \mathrm{~mm}$ linear travel allows the sample to be moved between the two sections of the surface chamber. The lower level is also equipped with a pair of laser view ports and an ion collection system followed by a low resolution time-offlight mass spectrometer with a microchannel plate (MCP) detector.

A major improvement to the new instrument is the short distance from nozzle to surface, only $180 \mathrm{~mm}$. This is to be compared to our previous instrument with $350 \mathrm{~mm}$ flight distance from nozzle to surface or the instrument of Schmid et al. at the EPFL in Lausanne, Switzerland with a nozzlesurface distance of $215 \mathrm{~mm}$. $^{9}$ This short distance required great care in the design and construction of the pumping chambers; however, shortening the flight distances provides numerous advantages including more intense molecular beams and shorter molecular beam pulses with the resulting ability to time resolve the scattered signal from the incoming beam background.

A schematic view of the molecule-surface scattering experiment is shown in Fig. 2. Pulsed molecular beams are generated by expanding through a pulsed nozzle. The expanded skimmed molecular beam passes through the first differential pumping stage, the (opened) slide gate valve, the second differential pumping stage, and an aperture into the UHV surface chamber where it collides with a metal surface and may then bounce back. Vibrationally excited molecules are prepared by laser overtone pumping, typically just $1-2 \mathrm{~cm}$ before the molecules encounter the surface in the 


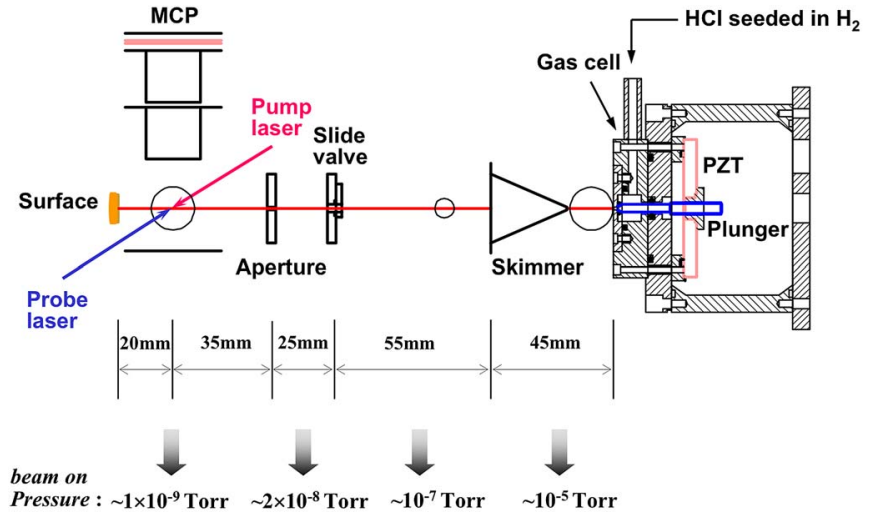

FIG. 2. (Color online) Schematic view of the molecule-surface scattering experiment. The distance from the nozzle to the surface is $180 \mathrm{~mm}$. Both incident and scattered molecules are probed $20 \mathrm{~mm}$ from surface. Laser preparing excited molecules can be performed upstream before or after the skimmer or downstream near to the surface. Optical access shown as circles. MCP: microchannel plate; PZT: piezoelectric translator.

UHV chamber. The incident and scattered molecules are probed by laser resonance enhanced multiphoton ionization (REMPI). The following sections go into greater detail.

\section{B. Molecular beam source}

\section{Pulsed source design}

Short molecular beam pulses are generated by expanding an inert carrier gas (typically $\mathrm{H}_{2}$ ) seeded with the sample molecules at $\sim 3$ atm stagnation pressure. The first experiments have been carried out using $\mathrm{HCl}$ as a sample gas. This points out one important aspect of the instrument: it is useful for corrosive samples. To accomplish this, we constructed the pulsed valve in a special way shown in Fig. 2. The pulsed valve is a modification of a standard HV piezoelectric translator (PZT) actuated pulsed valve, which borrows ideas from Dong et al.'s design. ${ }^{25}$ An extended cell is attached to a PZT (PI, P288.23) pulsed valve $(10 \mathrm{~Hz})$ to act as a gas holder and isolated from the PZT crystal by dynamically sealed O-ring so that the crystal is not exposed to the corrosive gases. The compression of the O-ring around the valve plunger needed to be carefully optimized to permit smooth motion, fast opening times and still provide dynamical sealing. The expansion orifice of the nozzle is $1 \mathrm{~mm}$ in diameter.

Remarkably short beam pulses could be produced by careful adjustment of the nozzle. Figure 3 shows the temporal profiles of seeded molecular beams of $1 \%$ and $10 \% \mathrm{HCl}$ in $\mathrm{H}_{2}$ measured $160 \mathrm{~mm}$ from the exit of the pulsed nozzle. Fitting these temporal profiles with Gaussians gives full width at half maximum (FWHM) values of 36 and $45 \mu \mathrm{s}$, respectively, with a minor bounce upon the closing of the valve. The spreading of the slower (10\%) in comparison to the faster (1\%) beam is due to the velocity distribution of the molecular beam, and one should note that the shorter pulse duration is expected to reflect more accurately the opening time of the pulsed valve. The short beam pulses $(<50 \mu \mathrm{s}$ FWHM) and the carefully designed short distance from nozzle to surface $(180 \mathrm{~mm})$ provides an intense molecular beam and the ability (to some degree) to resolve in time the incoming and outgoing (scattered) molecules.

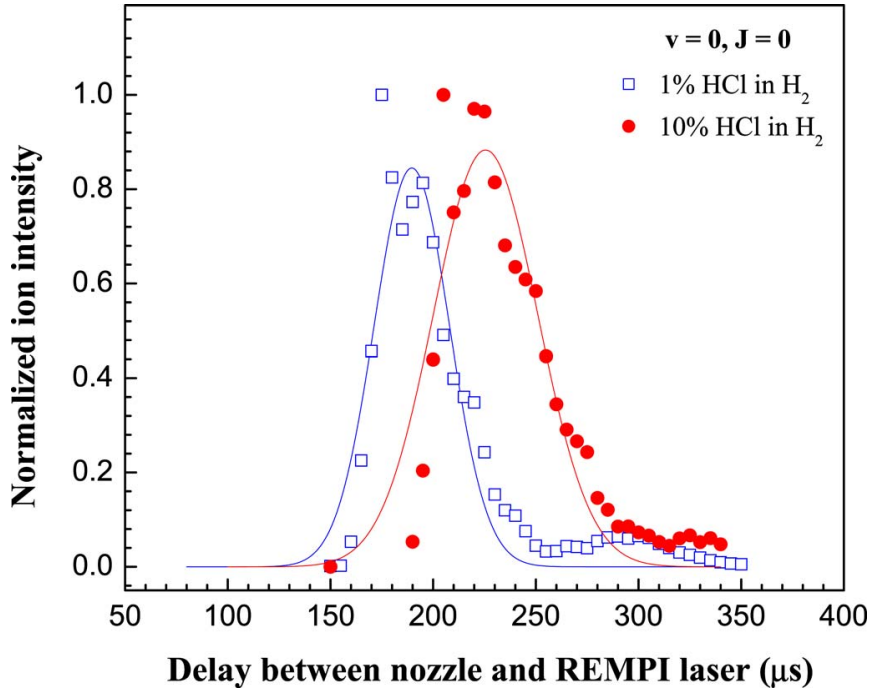

FIG. 3. (Color online) Temporal profiles of the pulsed molecular beam measured $\sim 160 \mathrm{~mm}$ downstream from the exit of the pulsed nozzle and without the surface present. Gaussian fits to the pulse indicate FWHM values of $35-50 \mu \mathrm{s}$.

High-density, small-divergence beams are achieved by careful adjustment of the nozzle and by proper optimization of the nozzle-skimmer alignment using a fast ion gauge. In our $\mathrm{HCl}$ experiments after a $1.5 \mathrm{~mm}$ diameter skimmer, followed by a $3 \mathrm{~mm}$ diameter aperture, and a $2 \mathrm{~mm}$ diameter aperture, the beam vertical height is $\sim 3 \mathrm{~mm}$ FWHM at a distance of $160 \mathrm{~mm}$ downstream from the orifice of the nozzle, meaning an angular divergence of about $0.7^{\circ}$.

The pulsed valve design also provides the capability to vary the incidence energy of translation over a reasonably wide range. For an ideal gas mixture, the most probable velocity of a supersonic beam is given by

$$
\bar{V}_{\infty}=\sqrt{\frac{2 \bar{C}_{P}}{\bar{M}} T_{0}},
$$

where $T_{0}$ is the stagnation gas temperature, $\bar{C}_{p}=\Sigma X_{i} C_{P i}$ and $\bar{M}=\Sigma X_{i} M_{i}$ are the molar average heat capacity and molar average molecular weight, respectively, and $X_{i}$ is the mole fraction. Thus, the kinetic energy of molecular beam can be varied easily over a broad range by seeding in either different carrier gases or by using different mixing ratios. For example, seeding $1 \%, 2.5 \%, 5 \%$, and $10 \% \mathrm{HCl}$ in $\mathrm{H}_{2}$ at $T_{0}$ $=298 \mathrm{~K}$ yields beam translational energies of $1.37,1.12$, 0.86 , and $0.59 \mathrm{eV}$, respectively. Our instrument can be used to measure the beam velocity by a two pulsed laser technique, where overtone pumping molecules between the nozzle and the skimmer in source chamber or between the skimmer and the slide valve in first differential pumping region, is used to "tag" a portion of the beam and state-specific REMPI detection can be performed downstream in surface chamber.

\section{Laser prepared molecules}

To investigate energy transfer in molecule-surface collisions, the initial and final quantum states of the scattering molecule should be determined by spectroscopic means. 


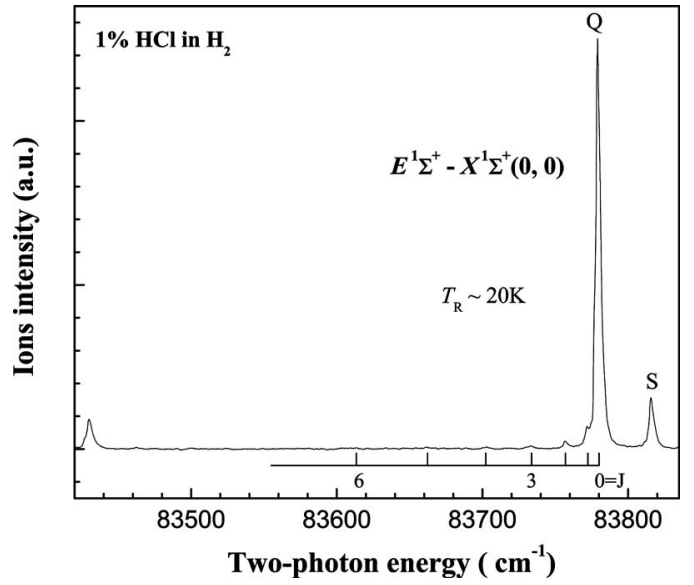

FIG. 4. $(2+1)$ REMPI spectrum of $\mathrm{HCl} v=0$ beam observed $\sim 160 \mathrm{~mm}$ downstream from the nozzle. Rotational temperature of the supersonic molecular beam is $20 \pm 10 \mathrm{~K}$.

REMPI is an ideal way to analyze the quantum state population distribution of the sample gas in the molecular beam. For example, vibrational ground state molecules can be produced directly by supersonic expansion of gas mixtures at room temperature. Figure 4 shows an example of the REMPI spectrum of $\mathrm{HCl}(v=0)$ prepared by expansion of $1 \% \mathrm{HCl}$ in $\mathrm{H}_{2}$. The rotational temperature of the beam is less than $20 \mathrm{~K}$, meaning that about $90 \%$ of population is in $J=0, v=0$.

Laser optical pumping through overtone transitions can be used to prepare molecules in specific initial vibrationally excited states. To accomplish this, we designed and built a difference frequency generation (DFG) setup to produce tunable IR light with narrow bandwidth and high pulse energies. A schematic of our DFG laser setup is displayed in Fig. 5. An injection seeded $Q$-switched Nd:YAG laser (YAG denotes yttrium aluminum garnet) (Continuum, PL7010) is frequency doubled to pump a tunable dye laser (Sirah, CSTRLG-18). The residual $1064 \mathrm{~nm}$ fundamental output from the same YAG laser is employed in an optical difference frequency mixing as the "signal wave," where the tunable output of the dye laser serves as the "pump wave." The two colors are combined with a dichroic mirror and carefully

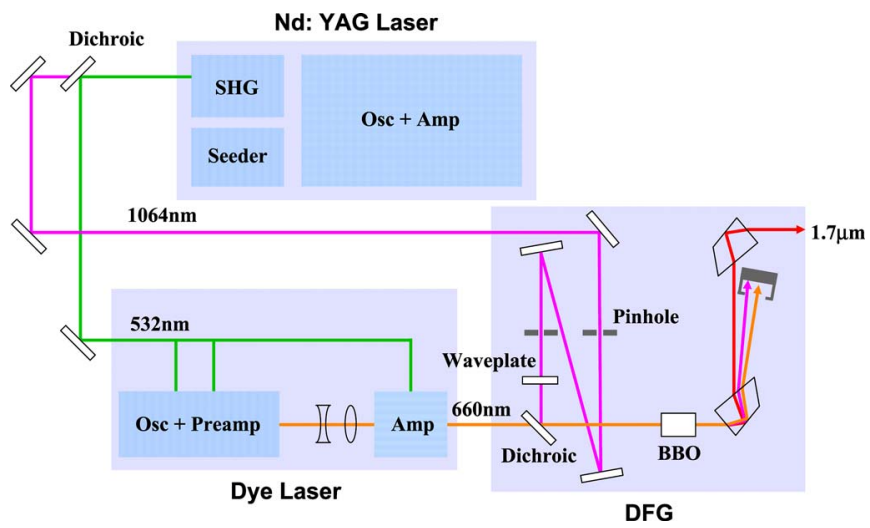

FIG. 5. (Color online) Schematic of the IR-DFG laser system used for the specific quantum state molecules preparation. Tunable, narrow-band IR light is produced by difference frequency mixing of a single-mode Nd:YAG $1064 \mathrm{~nm}$ laser and a narrow bandwith tunable dye laser. The quantum efficiency of IR DFG output can reach $40 \%$.

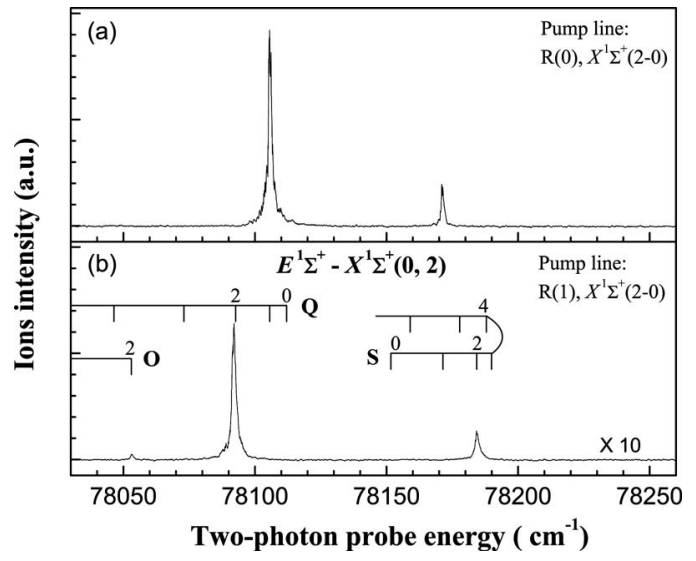

FIG. 6. $(2+1)$ REMPI spectra of a $\mathrm{HCl} v=2$ beam. The $v=2$ beam is prepared by laser overtone pumping of jet-cooled $10 \% \mathrm{HCl}$ in $\mathrm{H}_{2}$ beam. Overtone pumped and REMPI probed $\sim 160 \mathrm{~mm}$ downstream from the nozzle. (a) Prepared $v=2, J=1$ beam and laser pumped at $R(0)$ of $X^{1} \Sigma^{+}(2 \longleftarrow 0)$. (b) Prepared $v=2, J=2$ beam and laser pumped at $R(1)$ of $X^{1} \Sigma^{+}(2 \leftarrow 0)$.

overlapped in a $8 \times 8 \times 12 \mathrm{~mm}^{3} \beta$-barium borate $(\mathrm{BBO})$ crystal (Castech, type I, $p$-coated, $\theta=21^{\circ}$ ) to produce IR radiation at $1.5-1.9 \mu \mathrm{m}$ in the "idler wave." The polarizations of the input $1064 \mathrm{~nm}$ and the dye laser beams are horizontal and vertical, respectively; while the BBO crystal is mounted with its ordinary axis horizontal on a precision rotation stage for adjustment of the phase matching angle around an axis perpendicular to the direction of beam propagation in the horizontal plane. The generated IR light is also horizontally polarized. Two BK-7 Pellin-Broca prisms are used to separate IR output from the dye and $1064 \mathrm{~nm}$ waves. With this arrangement, we have successfully generated intense narrowband pulsed $\sim 1.7 \mu \mathrm{m}$ light, capable of saturating the $\operatorname{HCl}(v=2-0)$ transition. For these experiments the dye laser was operating on a dye mixture of DCM and LDS698 in methanol. Up to $13 \mathrm{~mJ} /$ pulse, $\sim 1.76 \mu \mathrm{m}$ IR radiation $\left(\sim 0.05 \mathrm{~cm}^{-1}\right.$ linewidth) was generated from $75 \mathrm{~mJ}$ of singlemode $1064 \mathrm{~nm}$ light with $60 \mathrm{~mJ}$ of $\sim 663 \mathrm{~nm}$ dye laser light. We find that the quantum efficiency

$$
\varepsilon=\frac{N_{\mathrm{IR}}}{\sqrt{N_{1064} N_{\mathrm{dye}}}},
$$

where $N$ refers to photon numbers in the three light pulses, is as high as $40 \%$. This is easily enough spectral brightness to saturate the $\operatorname{HCl}(v=0 \rightarrow 2)$ overtone transition. As nearly $90 \%$ of the $\mathrm{HCl}(v=0)$ molecules are in the $v=0, J=0$ state in the expansion-cooled beam, we are able to move a large fraction of the $\mathrm{HCl}$ molecules in the beam to $v=2$. Figure 6 shows two examples of the REMPI spectra for a beam of $\operatorname{HCl}(v=2)$ molecules, produced from the two dominantly populated rotational states in the beam. In panel (a), where $\operatorname{HCl}(v=2, J=1)$ was prepared, scanning the REMPI laser reveals the $Q(1)$ and weaker $S(1)$ lines only. In panel (b), where $\operatorname{HCl}(v=2, J=2)$ was prepared, $S(2), Q(2)$, and $O(2)$ can be seen. The spectrum in panel (b) is about ten times weaker as the population of the initial state, $\operatorname{HCl}(v=0, J=1)$ and is about ten times smaller than that of $\mathrm{HCl}(v=0, J=0)$. 

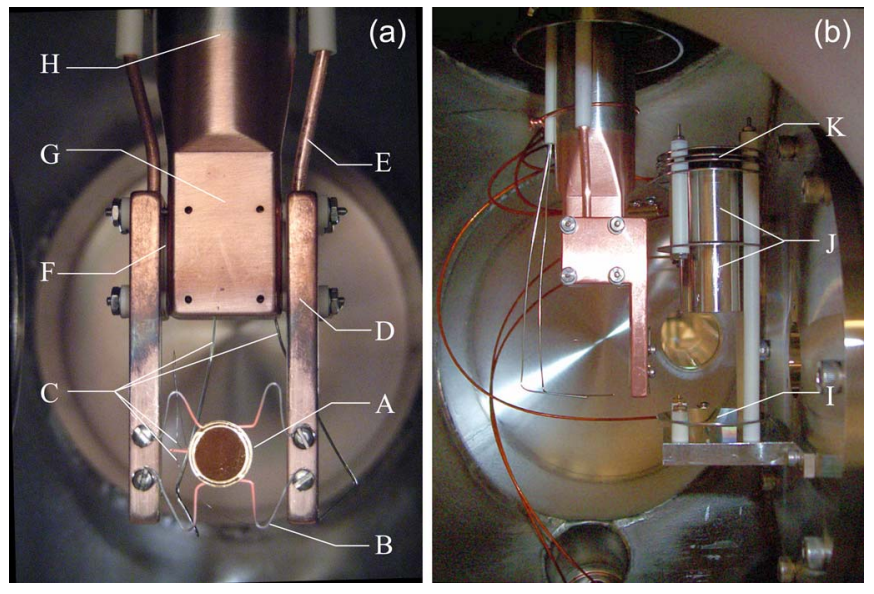

FIG. 7. (Color online) Photographs of the sample holder and the ion detector. (a) Surface sample holder: (A) Au(111) crystal (hot); (B) tungsten wire; (C) Chromel-Alumel thermocouple wires; (D) copper block; (E) electrical feedthrough rod; (F) sapphire spacer; (G) copper cold finger; and (H) stainless steel liquid nitrogen cryotube. (b). REMPI ion collection-detector system (stainless steel with $\mathrm{Al}_{2} \mathrm{O}_{3}$ ceramic posts) with sample holder (copper assembly). The molecular beam enters from the right. The view is along the propagation direction of the REMPI laser beam. Ion collection system consists of a repeller (I) and two cylindrical focusing lenses (J). Ions are detected by a MCP detector $(\mathrm{K})$.

\section{Target surface}

The sample surface is a $\mathrm{Au}(111)$ single crystal (Monocrystal, $10 \mathrm{~mm}$ diameter, $2 \mathrm{~mm}$ thick). It is mounted on a commercial high precision $X Y Z \Theta$ four-motion manipulator through a home-designed $\sim 1.45 \mathrm{~m}$ long sample holder, providing $800 \mathrm{~mm}$ translation along the vertical $Z$ axis, $\pm 25 \mathrm{~mm}$ $X$ and $Y$ translations in the horizontal plane (with $0.5 \mu \mathrm{m}$ precision), and $360^{\circ}$ rotation around the $Z$ axis. These four motions are used to move and orient the sample to different positions for molecular beam scattering, surface cleaning, and analysis. The $\mathrm{Au}(111)$ sample is mounted as shown in Fig. 7(a). It can be heated resistively using preshaped tungsten wires $(0.375 \mathrm{~mm}$ diameter $)$ pinched through two slots cut in the top and bottom sides of the sample and fixed on two copper blocks by screws. Two copper electrical feedthrough rods (0.094 in. diameter), which carried current from a dc power supply (Electronic Measurement, EMS7.5130) to the sample for heating, are mounted to the two copper blocks, respectively. Through a thin sapphire circular spacer (Swiss Jewel, W15.50), the copper blocks are contacted with a copper cold finger, which is welded together with a 1 in. diameter stainless steel liquid nitrogen cryotube. Here, sapphire spacers are used to limit thermal conduction at high temperatures and provide good thermal conduction at low temperatures, ${ }^{7}$ thereby facilitating resistive sample heating and conductive cooling, as well as providing electrical insulation. A Chromel-Alumel ( $K$-type) thermocouple wire is inserted into a small hole drilled on the side of the $\mathrm{Au}(111)$ crystal and is used for monitoring the surface temperature. To achieve accurate and reproducible surface temperature, we use a home-designed computer-based proportional-integral-derivative (PID) controller implemented in LabVIEW ${ }^{\mathrm{TM}}$. In our setup, any desired surface temperature from as low as $\sim 82 \mathrm{~K}$ to near the melting point of the metal sample can be easily obtained and stabilized

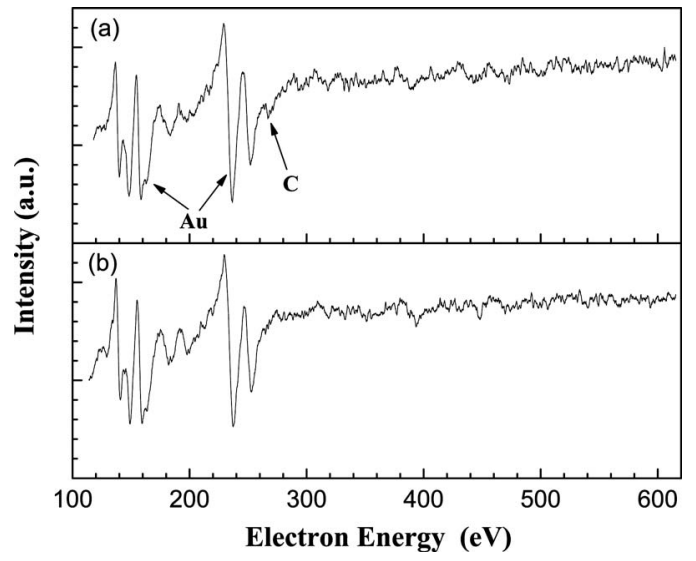

FIG. 8. Auger electron spectroscopy of the Au(111) surface in the UHV chamber: (a) before $\mathrm{Ar}^{+}$gun sputtering and (b) after sputtering. The main contamination on $\mathrm{Au}$ is carbon and it can be cleaned by sputtering.

$(< \pm 0.3 \mathrm{~K})$ within a few minutes. This provides us with a wide range of control over the sample surface temperature, which is critical to the investigation of electronically nonadiabatic influences.

The commercial Au(111) single crystal was prepared using standard sputter-anneal cycles in the UHV chamber until no contaminations could be detected by Auger electron spectrometer. It was cleaned daily by sputtering $\left(\sim 10 \mu \mathrm{A} / \mathrm{cm}^{2}\right)$ with $3 \mathrm{kV} \mathrm{Ar}{ }^{+}$for $15-20 \mathrm{~min}$, followed by annealing at $780 \mathrm{~K}$ for $20 \mathrm{~min}$ every $2-3 \mathrm{~h}$ to keep well-ordered (111) pattern. Auger electron spectroscopy (AES) was used to monitor surface cleanliness. Figure 8 shows the typical AES of $\mathrm{Au}(111)$ surface before and after sputtering and annealing cycles. The main impurity is carbon which can be easily removed by sputtering.

\section{Laser based molecular detection}

We employ the REMPI technique as a sensitive stateselective detection technique, applicable to a relatively large number of molecules. Here, $\mathrm{HCl}(v=0,1,2)$ molecules are probed using $(2+1)$ REMPI to selectively ionize specific quantum states via two-photon resonances from $X^{1} \Sigma^{+}\left(v^{\prime \prime}\right.$ $=0,1,2)$ to $E^{1} \Sigma^{+}\left(v^{\prime}=0\right)$ states near 239,247 , and $256 \mathrm{~nm}$, respectively. This was accomplished using a $355 \mathrm{~nm}$ YAG laser (Continuum, PL7010) to pump a dye laser (Sirah, CSTR-LG-24, $0.05 \mathrm{~cm}^{-1}$ linewidth), the output of which was doubled in BBO (Castech, $p$-coated, $\theta=55^{\circ}, 8 \times 8$ $\times 6.5 \mathrm{~mm}^{3}$ ) installed in a tracking system (Inrad, Autotracker II). We are able to produce $\sim 8 \mathrm{~mJ} /$ pulse of tunable laser light around $250 \mathrm{~nm}$. This UV light is focused with a $30 \mathrm{~cm}$ focal length lens, vertically translatable to measure scattering angular distributions. The ion time-of-flight collection system has also been carefully designed [See Fig. 7(b)] and consists of two cylindrical focusing lenses in addition to an ion repeller with a $6.5 \mathrm{~cm}$ ion flight distance to the MCP detector. This provides sufficient mass resolution (for example $\mathrm{H}^{+}$can easily be resolved from $\mathrm{HCl}^{+}$and $\mathrm{Cl}^{+}$in $\mathrm{HCl}$ REMPI), while still providing unit mass collection efficiency. This design allows us to evaluate possible stateselective influences on dissociative ionization that might oth- 


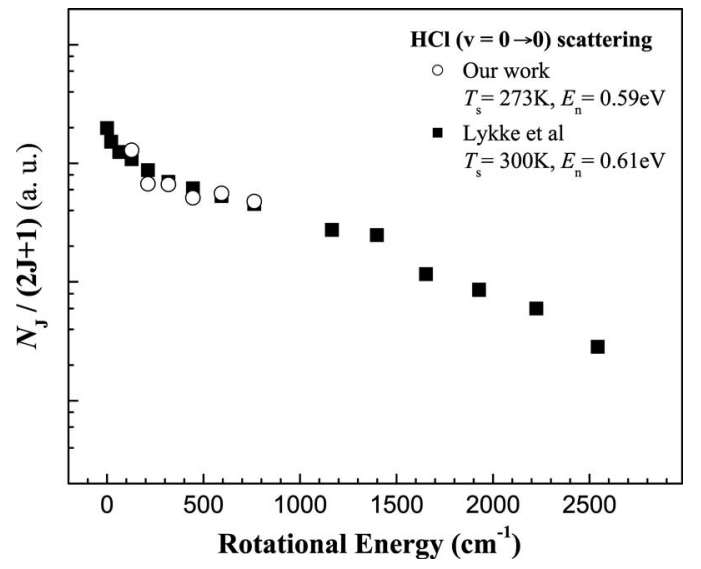

FIG. 9. A comparison of the rotational Boltzmann distributions for $\mathrm{HCl}(v$ $=0)$ scattered from $\mathrm{Au}(111)$ between Lykke and Kay's data (Ref. 26) and this work. $E_{n}$ is the incidence energy in the surface normal plane, defined as $E_{n}=E_{i} \cos ^{2}\left(\Theta_{i}\right)$, where $\Theta_{i}$ is the incidence angle relative to surface normal. $\Theta_{i}$ is $45^{\circ}$ and $3.3^{\circ}$ for Lykke and Kay's and our experiments, respectively. The results are in a good agreement. Our $J=0-2$ data are not shown due to the incoming beam background at low $J$.

erwise influence our measurements of rotationally resolved signal strengths.

The integrated ion intensity was recorded. The incident and scattered molecules were both detected $20 \mathrm{~mm}$ from the surface at different delay times $(20-30 \mu \mathrm{s})$. Our design permits the beam measurements in a distance of $5-35 \mathrm{~mm}$ from the surface. Vibrational-rotational distributions were recorded by scanning the probe (REMPI) laser frequency. Angular distributions were measured by translating the probe laser beam along a line perpendicular to the molecular beam direction. Temporal profiles may be obtained by recording the intensity of the REMPI signal as a function of the time delay between the probe laser and opening time of the pulsed valve.

\section{PERFORMACE OF THE APPARATUS}

This section presents some preliminary experimental results that demonstrate some of the performance characteristics of the new instrument. Our new instrument is designed to examine inelastic scattering of molecules colliding at solids. We have recently explored the scattering of $\operatorname{HCl}(v=0)$ on $\mathrm{Au}(111) .^{23,24}$

\section{A. Rovibrational distributions}

We have obtained large quantities of high quality data on the vibrationally elastic scattering of $\operatorname{HCl}(v=0 \rightarrow 0)$ from $\mathrm{Au}(111)$ including variations of the angular and rotational distributions with surface temperature and incidence energy. These results are in a good agreement with the previous work by Lykke and $\mathrm{Kay}^{26}$ and provide a solid validation of the reliability of the data produced by the new apparatus. A comparison of the previous data obtained by Lykke and $\mathrm{Kay}^{26}$ to our result is shown in Fig. 9. The agreement is good.

Figure 10 displays representative $(2+1)$ REMPI spectra for the incident supersonic molecular beam containing $1 \%$ $\mathrm{HCl}$ in $\mathrm{H}_{2}\left(E_{i}=1.37 \mathrm{eV}\right)$ and the molecular beam scattered

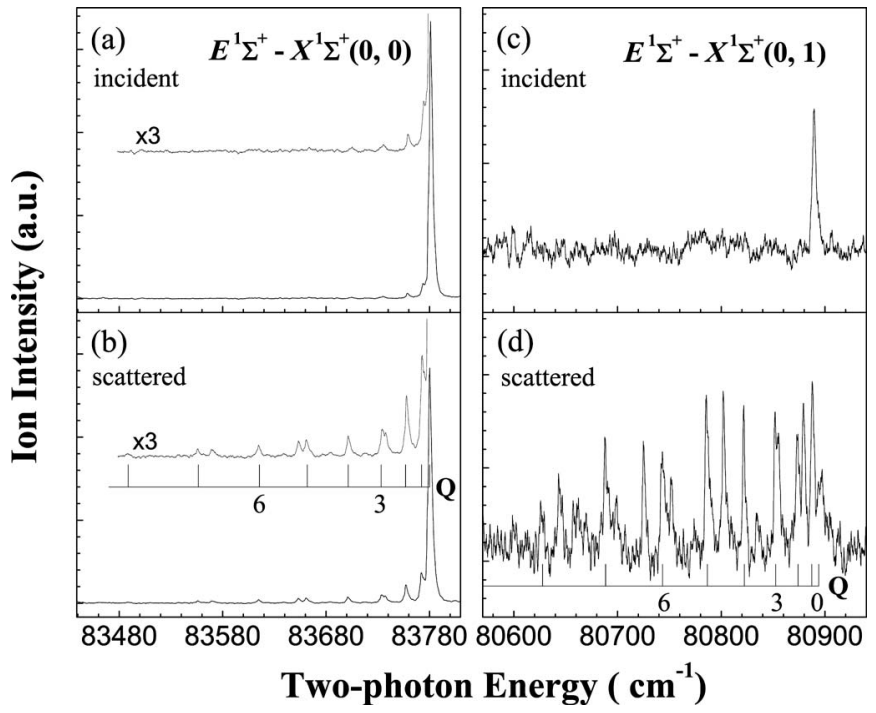

FIG. 10. Representative $(2+1)$ REMPI spectra of the incident supersonic molecular beam $\left(1 \% \mathrm{HCl}\right.$ in $\left.\mathrm{H}_{2}\right)$ and the molecular beam scattered from a $1073 \mathrm{~K} \mathrm{Au}(111)$ surface. [(a) and (b)] $\mathrm{HCl}(v=0 \rightarrow 0)$ rotational excitation. $[(\mathrm{c})$ and $(\mathrm{d})] \mathrm{HCl}(v=0 \rightarrow 1)$ vibrational excitation. The incident and scattered signals are measured at a $20 \mathrm{~mm}$ distance from the surface along the incoming beam direction ( $3.3^{\circ}$ away from the surface normal) with $20 \mu \mathrm{s}$ delay time. The $v=0$ and $v=1$ signals are measured at different MCP voltages, i.e., 1.56 and $1.70 \mathrm{kV}$, respectively. The high sensitivity of our instrument can be seen.

from a clean $1073 \mathrm{~K} \mathrm{Au}(111)$ surface. Figures 10 (a) and 10(b) show raw REMPI spectra reflecting rotationally inelastic scattering. Panel (a) shows the incoming beam before interaction with the $\mathrm{Au}(111)$ surface, while panel (b) shows the spectrum obtained at a $20 \mu$ s later time after the opening of the pulsed valve, where outgoing scattered molecules are detected. REMPI spectra are obtained using the two-photon $E(0)-X(0)$ vibronic band. We are also easily able to detect $\mathrm{HCl}$ in $v=1$ using the $E(0)-X(1)$ vibronic band [Fig. 10(c)]. Note the low rotational temperature $\left(T_{R} \sim 20 \pm 10 \mathrm{~K}\right)$ indicated by the small number of lines in the incoming beam spectra. Thermally populated $\mathrm{HCl}(v=1)$ can clearly be seen in the molecular beam even though the thermal population of $\operatorname{HCl}(v=1)$ at the stagnation pressure of the expansion $(300 \mathrm{~K})$ is only $9.8 \times 10^{-7}$. Notably, these signals can be used as a means of calibrating the absolute vibrational excitation probabilities due to surface scattering. Figure 10(d) shows the assigned $Q$ branch up to $J=8$ lines for scattered $\operatorname{HCl}(v=1)$.

\section{B. Temporal distributions}

The results just presented show us that the incoming beam is predominantly found in $v=0, J=0$ and 1 , whereas the scattered, outgoing beam is populated in higher energy quantum states. The temporal distributions of incident and scattered molecules were recorded for a beam of $\mathrm{HCl}$ molecules at $E_{i}=1.37 \mathrm{eV}$ and are displayed in Fig. 11. Here, we may probe $\operatorname{HCl}(v=0, J=0)$ with the surface moved away from the molecular beam and record the temporal distribution of the incident beam (shown as closed circles). When the REMPI laser probes higher $J$ states, we may record the temporal distribution of the scattered molecules (shown as 


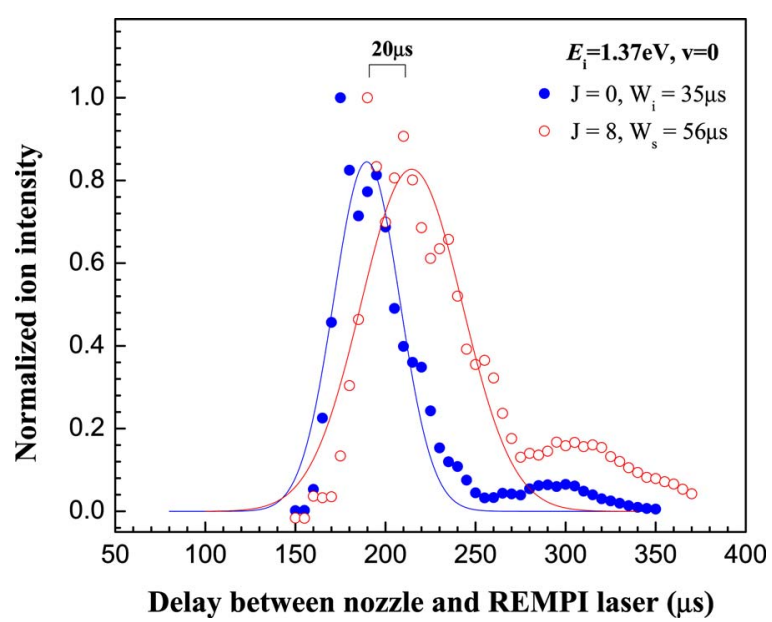

FIG. 11. (Color online) Temporal profiles of the incident and scattered $\mathrm{HCl}$ molecular beams at $E_{i}=1.37 \mathrm{eV}$. Both are measured $\sim 160 \mathrm{~mm}$ downstream from the nozzle. The incident beam was recorded without the surface present.

open circles). The two pulses have both been normalized to their maxima. The time shift we see is quite helpful in discriminating against background and is only possible due to the remarkably short pulse duration of the pulsed valve we are using.

\section{Angular distributions}

The instrument may also be used to record angular distributions of scattered molecules for molecular beams at near normal incidence. Representative angular distributions are displayed in Fig. 12, for scattered $\operatorname{HCl}(v=1, J=6)$ at $E_{i}$ $=1.12 \mathrm{eV}$ and several surface temperatures. For reference, the angular distribution of the incoming molecular beam is also shown. All the data have been fit with Gaussian distributions, shown as solid lines in the figure. The angular distributions of the scattered molecules all peak at $\sim 6.5^{\circ}$ angle

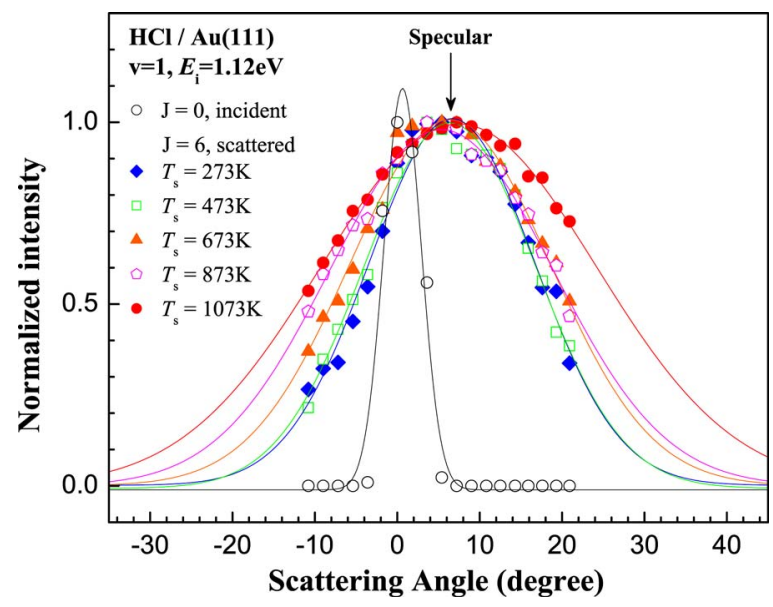

FIG. 12. (Color online) Angular distributions for vibrationally inelastic scattering of $\mathrm{HCl}(v=0 \rightarrow 1, J=6)$ from $\mathrm{Au}(111)$ at $E_{i}=1.12 \mathrm{eV}$. For reference, angular distribution of the incoming molecular beam is also shown. The points are the experimental data, while the solid lines are Gaussian fits to the data. Note that the scattered beams peak slightly away from $0^{\circ}$, the nominal surface normal, due to the fact that the molecular beam is incident $\sim 3.3^{\circ}$ off normal. We estimate that out-of-plane ionization is important over $\sim \pm 30^{\circ}$ with respect to the surface normal, along the laser propagation direction.

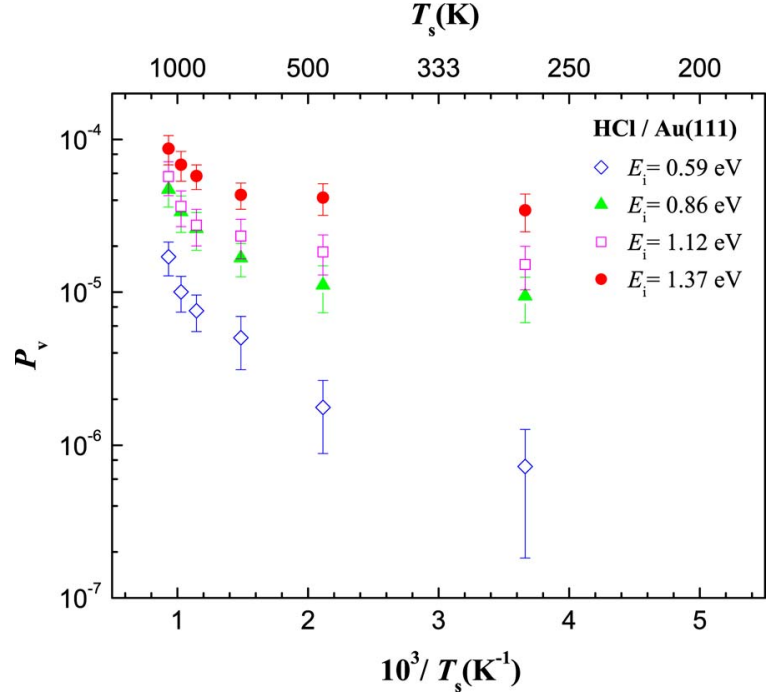

FIG. 13. (Color online) Surface temperature dependence of vibrational excitation probability of $\mathrm{HCl}(v=0 \rightarrow 1)$ from $\mathrm{Au}(111)$ at four incidence energies and six surface temperatures. $P_{v}$ 's are in the range of $10^{-4}-10^{-6}$.

(relative to the incoming beam axis). To measure the absolute angle between the molecular beam axis and the surface normal, a He-Ne laser was temporarily installed at the position of the pulsed valve and was aligned to pass through the skimmer and apertures, whereby it would shine onto the surface. The reflection angle of the laser light shows that our surface is slightly $\left(3.25^{\circ} \pm 0.30^{\circ}\right)$ off-normal incidence of the incoming molecular beam. Thus, the observed $6.5^{\circ}$ peak in the scattered molecule angular distribution is exactly appearing at the specular direction. As can be seen in this figure, angular distributions of scattered $\operatorname{HCl}(v=1)$ are all quasi-specular, which is indicative of the direct-inelastic scattering, and broaden gradually in width with increasing surface temperature due to the surface-atom thermal motion. ${ }^{27}$

\section{Vibrational excitation probability}

As it has been recently reported, ${ }^{24}$ this instrument has been used to obtain extensive data on vibrationally inelastic scattering of $\mathrm{HCl}(v=0 \rightarrow 1)$, including rotational state distributions as well as state-selected angular and temporal distributions under 24 different collisional conditions: six surface temperatures $T_{s}=273,473,673,873,973$, and $1073 \mathrm{~K}$ and four translational incidence energies $E_{i}=1.37,1.12,0.86$, and $0.59 \mathrm{eV}$. By summing all the observed $v=1$ rotational line intensity [in data such as that shown in Fig. 10(d)], we may compare to the thermal $v=1$ population in the incident molecular beam [in data such as Fig. 10(c)], and, after correcting for differences in rotational line-strength, laser intensity, angular, and temporal distributions, we are able to estimate the absolute $\mathrm{v}=0 \rightarrow 1$ excitation probabilities. ${ }^{23}$ Figure 13 shows the full surface temperature $T_{s}$ dependence of the derived vibrational excitation probability of $\mathrm{HCl}(v=0 \rightarrow 1)$ on $\mathrm{Au}(111)$ at four incidence energies. Here, we measured the lowest excitation probability for $\mathrm{HCl}$ on $\mathrm{Au}$ to be about 8 $\times 10^{-7}$, which is about 100000 times smaller than the largest excitation probabilities found in previous experiments (em- 
ploying $(1+1)$ REMPI) on NO carried out in similar instruments. We estimate that vibrational excitation probabilities as low as $\sim 10^{-7}$ are still measurable with this apparatus.

\section{SUMMARY}

We have designed and constructed an advanced molecular beam-surface scattering apparatus for the purpose of beam-surface energy transfer studies. The performance of this instrument has been demonstrated by vibrational excitation studies of $\mathrm{HCl}(v=0 \rightarrow 1)$ on $\mathrm{Au}(111)$. This apparatus improves on previous instruments in several ways, including design and implementation of the molecular beam source, improved detection sensitivity due to improved beam intensity, improved time resolution, and improved optical pumping efficiency. Using $(2+1)$ REMPI detection for $\mathrm{HCl}$, we can observe vibrationally inelastic events that are $10^{5}$ times smaller than previously reported NO results employing (1 +1) REMPI. With such high sensitivity, one expects that this instrument can provide similar data on a wide variety of molecule-surface scattering combinations.

\section{ACKNOWLEDGMENTS}

We greatly appreciate the financial support from DOE Office of Basic Energy Sciences Grant No. DE-FG0203ER1544 and Partnership for International Research and Education-for Electronic Chemistry and Catalysis at Interfaces-NSF Grant No. OISE-0530268. We gratefully thank Mr. Terry Hart and Mr. Bruce Dunson in the machine shop at Chemistry Department of UCSB for providing the excellent support.

${ }^{1}$ J. A. Barker and D. J. Auerbach, Surf. Sci. Rep. 4, 1 (1985).

${ }^{2}$ C. R. Arumainayagam and R. J. Madix, Prog. Surf. Sci. 38, 1 (1991).

${ }^{3}$ M. L. Yu and L. A. Delouise, Surf. Sci. Rep. 19, 289 (1994).

${ }^{4}$ R. B. Gerber, Chem. Rev. (Washington, D.C.) 87, 29 (1987).
${ }^{5}$ C. T. Rettner, D. J. Auerbach, J. C. Tully, and A. W. Kleyn, J. Phys. Chem. 100, 13021 (1996).

${ }^{6}$ A. M. Wodtke, J. C. Tully, and D. J. Auerbach, Int. Rev. Phys. Chem. 23, 513 (2004).

${ }^{7}$ F. Pradere, M. Chateau, M. Benslimane, M. Bierry, M. Chatelet, D. Clement, A. Guilbaud, J. C. Jeannot, A. Demartino, and H. Vach, Rev. Sci. Instrum. 65, 161 (1994).

${ }^{8}$ H. Yoshikawa, H. Ohashi, K. Tabayashi, M. Suzui, T. Horigome, K. Hayakawa, S. Kato, and K. Shobatake, Rev. Sci. Instrum. 70, 1806 (1999).

${ }^{9}$ M. P. Schmid, P. Maroni, R. D. Beck, and T. R. Rizzo, Rev. Sci. Instrum. 74, 4110 (2003).

${ }^{10}$ C. T. Rettner, F. Fabre, J. Kimman, and D. J. Auerbach, Phys. Rev. Lett. 55, 1904 (1985).

${ }^{11}$ B. D. Kay, T. D. Raymond, and M. E. Coltrin, Phys. Rev. Lett. 59, 2792 (1987).

${ }^{12}$ M. Morin, N. J. Levinos, and A. L. Harris, J. Chem. Phys. 96, 3950 (1992).

${ }^{13}$ Y. Huang, A. M. Wodtke, H. Hou, C. T. Rettner, and D. J. Auerbach, Phys. Rev. Lett. 84, 2985 (2000).

${ }^{14}$ C. T. Rettner, D. J. Auerbach, and H. A. Michelsen, Phys. Rev. Lett. 68, 2547 (1992).

${ }^{15}$ E. K. Watts, J. L. W. Siders, and G. O. Sitz, Surf. Sci. 374, 191 (1997).

${ }^{16}$ A. M. Wodtke, H. Yuhui, and D. J. Auerbach, Chem. Phys. Lett. 413, 326 (2005).

${ }^{17}$ L. C. Shackman and G. O. Sitz, J. Chem. Phys. 123, 064712 (2005).

${ }^{18}$ A. C. Luntz, M. Persson, and G. O. Sitz, J. Chem. Phys. 124, 091101 (2006).

${ }^{19}$ H. Hou, Y. Huang, S. J. Gulding, C. T. Rettner, D. J. Auerbach, and A. M. Wodtke, Science 284, 1647 (1999).

${ }^{20}$ Z. H. Liu, L. C. Feldman, N. H. Tolk, Z. Y. Zhang, and P. I. Cohen, Science 312, 1024 (2006).

${ }^{21}$ Y. H. Huang, C. T. Rettner, D. J. Auerbach, and A. M. Wodtke, Science 290, 111 (2000).

${ }^{22}$ J. D. White, J. Chen, D. Matsiev, D. J. Auerbach, and A. M. Wodtke, Nature (London) 433, 503 (2005).

${ }^{23}$ Q. Ran, D. Matsiev, D. J. Auerbach, and A. M. Wodtke, Nucl. Instrum. Methods Phys. Res. B 258, 1 (2007).

${ }^{24}$ Q. Ran, D. Matsiev, D. J. Auerbach, and A. M. Wodtke, Phys. Rev. Lett. 98, 237601 (2007).

${ }^{25}$ F. Dong, S. H. Lee, and K. Liu, J. Chem. Phys. 113, 3633 (2000).

${ }^{26}$ K. R. Lykke and B. D. Kay, J. Chem. Phys. 92, 2614 (1990).

${ }^{27}$ F. O. Goodman and H. Y. Wachman, Dynamics of Gas-Surface Scattering (Academic, New York, 1976). 